\title{
Use of a Novel Stool Scoring System for Assessing Bowel Habits and Laxatives use Amongst Older People-A Pilot Study in Kingston, Ontario
}

Rasika Wijeratne ${ }^{1,2}$ and Lawrence Leung ${ }^{1,2 *}$

${ }^{1}$ Department of Family Medicine, Queen's University, Canada

${ }^{2}$ Centre of Studies in Primary Care, Queen's University, Canada

\begin{abstract}
Background: Constipation is a common complaint in the care of elderly in long term care facility. The Bristol Stool Chart (BSC) has been the gold standard for describing the form and consistency of bowel motions but is limited as a descriptive qualitative tool.

Objective: To pilot the feasibility of a novel stool scoring system in quantitative analysis of bowel motions and their texture.

Methods: 100 medical records were randomly selected from a local long-term care facility to extract descriptions of bowel movements and stool textures in the previous 7 days. A novel numeric stool score of +3 to -3 for each documented bowel motion and a cumulative value was obtained. Such score was recorded against the total cost of treatment.

Results: In the period studied, there was no significant difference in the mean bowel motions, the cumulative stool score and costs of laxative treatment between the male and female population.
\end{abstract}

Keywords: Bowel motions; Stool scoring system

\section{Introduction}

\section{Problem of chronic constipation}

Chronic constipation is a common problem seen in the older people and long term care residents. The term constipation may be interpreted differently by patients as compared to physicians. While physicians often equate constipation with less than three bowel movements per week [1,2], patients would consider other factors like urge, straining, feelings of incomplete emptying and stool consistency $[3,4]$. Here in Kingston, Ontario, a therapeutic protocol for chronic constipation had been used in a long-term care facility amongst the residents. Each resident has a stool chart which documented the stool frequency and stool texture as per the Bristol Stool Chart (BSC), first described by Lewis and Heaton in 1997 [5]. In an attempt to evaluate the cost-effectiveness of the existing therapeutic protocol for chronic constipation, difficulty was confronted as the BSC classified stool texture in a qualitative way. Both the resident and care-providers could only arrive at a subjective consensus regarding overall efficacy of the treatment protocol. It would be logical to implement a numeric scoring system that define the stool texture which enables quantitative analysis both within the subjects and across subjects over a period of time.

\section{Objective}

To pilot test a numeric stool score for analyzing chronic constipation amongst the older people population and analyze the effectiveness of using different laxatives as per protocol in a selected long term care facility in Ontario.

\section{Materials and Methods}

\section{Design}

Cross-sectional retrospective study of recorded data regarding bowel motions and stool consistency of residents in a long-term care facility.

\section{Study population and sampling methods}

Prior to commencement of study, approval has been obtained from the Health Services Research Ethics Board (HSREB) of Queen's Faculty of Health Sciences and the Director of the long term care facility (Providence Manor). 100 medical records of residents who are alive and living which contained documented treatment of chronic constipation were randomly selected for review. There are no exclusion criteria. The participants' age and gender were recorded. In a retrospective cross-sectional manner, the stool charts of each medical record were reviewed for the seven days from 2011 Dec 22 to 2011 Dec 28.

\section{Data collection}

All records were anonymized at the Centre of Studies in Primary Care (CSPC), with an identity tracer kept confidentially at the Research Office repository. Data was extracted regarding the number of bowel movements and the stool consistency of each motion, which was already evaluated as per Bristol Stool Chart (BSC) [5]. The original BSC only categorizes stool consistency in a descriptive way from Types 1 (most constipated) to Type 7 (total diarrhea). A novel Stool Scoring System (SSS) is hence devised where a numeric value ranging from -3 to +3 is arbitrarily assigned to different types of stool formation (Figure 1). A stool score of +3 will correspond to BSC type 1 (rock-like stool), a stool score of -3 will correspond to BSC type 7 (total liquid diarrhea) and a stool score of 0 will refer to BSC type 4 (normal consistency).

*Corresponding author: Lawrence Leung, Centre of Studies in Primary Care, Queen's University, 220 Bagot Street, Kingston ON K7L 5E9, Canada, E-mail: leungl@queensu.ca

Received July 11, 2013; Accepted September 17, 2013; Published September 27,2013

Citation: Wijeratne R, Leung L (2013) Use of a Novel Stool Scoring System for Assessing Bowel Habits and Laxatives use Amongst Older People-A Pilot Study in Kingston, Ontario. J Gen Pract 1: 124. doi: 10.4172/2329-9126.1000124

Copyright: (c 2013 Wijeratne R, et al. This is an open-access article distributed under the terms of the Creative Commons Attribution License, which permits unrestricted use, distribution, and reproduction in any medium, provided the original author and source are credited. 


\begin{tabular}{|c|c|l|}
\hline Stool Score & Visual Appearance & Description \\
\hline+3 & & Individual hard pieces \\
\hline+2 & & $\begin{array}{l}\text { Single elongated firm piece } \\
\text { with knobbly surface }\end{array}$ \\
\hline+1 & & Single elongated semi- \\
\hline 0 & & Parm piece with fissures \\
\hline-1 & & Individual soft pieces \\
\hline-2 & & All liquid without pieces \\
\hline-3 & & \\
\hline
\end{tabular}

Figure 1: Numeric stool scoring system modified from the Bristol Stool Chart (BSC). Normal stool consistency (BSC type 4) is given a value 0 ; a value of of +3 indicates individual hard stools (BSC Type 1), whilst -3 indicates diarrhoeal state with totally liquid stool (BSC type 7). (Diagram drawn by Arthur Leung).

A numeric total, referred to as Cumulative Stool Score (CSS) is then obtained by summing the scores for all bowel motions within the seven days. This CSS score reflects the overall quality of bowel habit for each subject over the week. The stool chart of each subject is updated by the responsible charge nurse. Self-reported bowel movements that were not verified by the charge nurse were considered invalid, and any subject with one or more invalid bowel movements will not qualify for a cumulative stool score. Take for example, if the subject had 6 documented motions in the week which were all soft and elongated in one piece, CSS will be zero $(6 \times 0)$ which indicates normal consistency. In other words, a positive CSS will indicate passage of hard stools during the week while a negative CSS will indicate a diarrheal state. Moreover, the types and amount of laxatives (if any) given to each resident during that week were also recorded.

\section{Data analysis}

Data extracted were tabulated in Excel sheets and two-tailed MannWhitney $\mathrm{U}$ test is applied for analysis, assuming unequal variance between the two groups of male and female subjects. The SPSS $\odot 20.0$ package was used as the statistical analysis software program. Twotailed significance is used to test the null hypothesis that the distribution of a variable is the same between the male and female gender. The usual value of $\mathrm{p}<0.05$ is taken as statistically significant.

\section{Results}

Results of the selected 100 records were included in the initial stage of analysis. The demographic characteristics are given in Table 1.

Of the 100 subjects initially surveyed, 26 were males and 74 were females. Mean age was $83.6(\mathrm{SD}=10.9)$ with a difference in distribution which was found to be statistically significant. Overall (counting both reported and observed) mean bowel motions per week was 4.32 $(\mathrm{SD}=2.37)$ with no significant difference in the distribution between the male and female. $62 \%$ of subjects had laxatives ordered on a

\begin{tabular}{|c|c|c|c|c|c|}
\hline \multirow{2}{*}{\multicolumn{2}{|c|}{ Age }} & Mean & Std. Deviation & Minimum & Maximum \\
\hline & & 83.60 & 10.90 & 44 & 105 \\
\hline \multicolumn{2}{|c|}{$\begin{array}{l}\text { Number of bowel motions } \\
\text { (BM \#) }\end{array}$} & 4.32 & 2.37 & 1 & 14 \\
\hline \multicolumn{2}{|l|}{ Cost (in cents) } & 15.88 & 20.32 & .00 & 124.42 \\
\hline \multicolumn{6}{|c|}{ Total number of subjects=100 $(F=74, M=26)$} \\
\hline & \multicolumn{2}{|c|}{ Age } & \multicolumn{2}{|l|}{ BM\# } & Cost \\
\hline Mann-Whitney U & \multicolumn{2}{|c|}{437.500} & \multicolumn{2}{|l|}{932.500} & 928.500 \\
\hline $\mathbf{Z}$ & \multicolumn{2}{|c|}{-4.126} & \multicolumn{2}{|l|}{-.234} & -.266 \\
\hline Exact Sig. (2-tailed) & \multicolumn{2}{|c|}{.000} & \multicolumn{2}{|l|}{.818} & .793 \\
\hline
\end{tabular}

Test Statistics

Table 1: Data of 100 individuals counting all bowel motions (verified and selfreported).

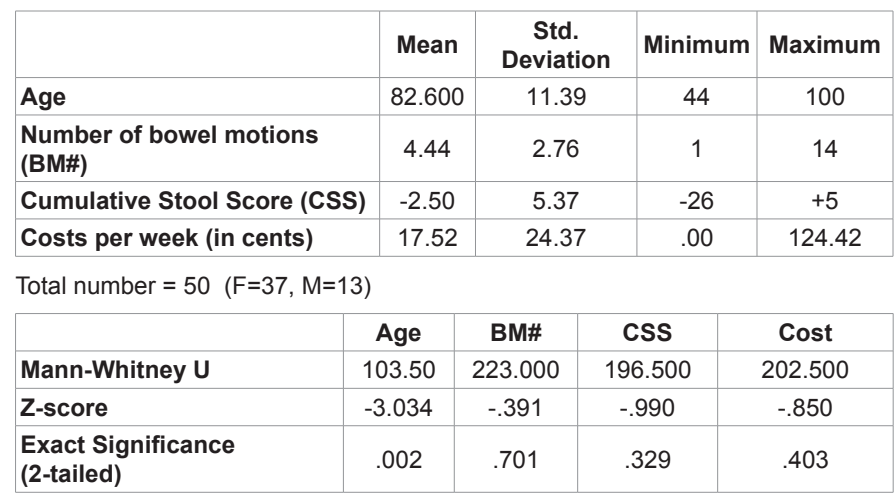

Test Statistics

Table 2: Data of 50 individuals counting verified bowel motions.

regular basis and $83 \%$ on "as required" basis. $78 \%$ of subjects received at least one laxative during the surveyed week. Senokot was the most frequently used laxative and the average subject consumed 1.37 units of Senokot during the surveyed week. Overall costs of laxatives per week per individual was 15.88 cents $(S D=20.32)$, again with no statistically significant difference between the male and female group.

As 210 out of 432 (48.6\%) bowel movements were not verified by the charge nurse, they were hence invalidated. As a result, only 50 subjects ( 13 male and 37 female) yielding 222 (51.4\%) bowel movements qualified for the CSS scoring and further analysis (Tables 2 and 3). Mean age was $82.6(\mathrm{SD}=11.39)$ with a distribution which was found to be statistically significant between the male and the female. Overall (counting both reported and observed) mean bowel motions per week was $4.44(\mathrm{SD}=2.76)$ with no significant difference in the distribution between the male and female. CSS was $-2.5(\mathrm{SD}=5.37)$ for the whole week which translated to an average daily score of -0.36 (which can be rounded off to normal stool consistency in the original Bristol Stool Score), with no significant difference between the two genders. Overall costs of laxatives per week per individual was 17.52 cents $(\mathrm{SD}=24.37)$. According to the Mann-Whitney mean rankings, apart from the age in female group which is significantly higher than the age in male group, there is no statistically significant difference in the distribution of total bowel motions, cumulative stool score and costs of laxatives per week between the female and the male group (Table 3).

\section{Discussion}

The prevalence of constipation in North America varied widely from $1.9 \%$ to $27.2 \%$ [6,7] with $50 \%$ to $74 \%$ of the institutionalized elderly reporting daily use of laxatives $[8,9]$. Females are two to three times more likely than males to suffer from constipation in terms 


\begin{tabular}{|l|l|l|l|l|}
\hline & Null Hypothesis & \multicolumn{1}{|c|}{ Test } & Sig & Decision \\
\hline $\mathbf{1}$ & The distribution of Age is the same across categories of Gender. & Independent- Samples Mann-Whitney U Test & .002 & Reject the null hypothesis. \\
\hline $\mathbf{2}$ & The distribution of BM\# is the same across categories of Gender. & Independent-Samples Mann-Whitney U Test & .696 & Retain the null hypothesis. \\
\hline $\mathbf{3}$ & The distribution of CSS is the same across categories of Gender. & Independent- Samples Mann-Whitney U Test & .322 & Retain the null hypothesis. \\
\hline $\mathbf{4}$ & The distribution of Cost is the same across categories of Gender. & Independent- Samples Mann-Whitney U Test & .395 & Retain the null hypothesis. \\
\hline
\end{tabular}

Hypothesis Test Summary

Asymptotic significances are displayed. The significance level is .05 .

Table 3: Summary showing conclusions regarding distribution of different variables between the female and male group.

of prevalence [3,6,10-12] and physical symptoms [6,11]. Advanced age also predisposes to chronic constipation with an abrupt rise in prevalence after the age of 70 years [6]. Possible causes would include decreased urge for defecation, medication and physical immobility. In United States, constipation ranks amongst the top five gastrointestinal reasons for out-patient visits [13]. Diagnostic workup for constipation averages US $\$ 3,000$ per year [14] and it takes another US\$ 4500 per year to provide treatment [15] for patient. Overall, chronic constipation has significant negative impact on patients' quality of life from various culture and nationalities [16], especially in the older people [17] .

Etiology of chronic constipation can be broadly divided into slow transit time and pelvic floor dysfunction, and various treatment options have been reviewed according to their evidence [18]. However, there is not much literature about analysis on the cost-effectiveness of these various treatments. This motivated the authors to carry out this study to pilot-test a novel stool scoring system in an attempt to better describe bowel habits data in a quantitative way.

Overall, our data supports the consensus that the average resident in the long-term care facility enjoyed a reasonable number of bowel movements per week ( 4.4 motions in a week) with overall acceptable stool consistency (near the normal consistency ranking). However, our study had a number of limitations. Firstly, about half of the recorded bowel movements were not visually verified by the staff nurse and hence were not rated as per the Bristol Stool Score. Anyone having more than two unverified bowel motions was also excluded from the final analysis. Second, it was assumed that the numbers of bowel movements not seen by the nursing staff were self-reported accurately by the resident, and this may be prone to errors given the prevalence of cognitive impairment amongst the population in the care facility. Moreover, co-morbid conditions and current medications of the subjects could not be detailed from the individual charts which might be relevant to the study. Finally, our study was a pilot conducted in one institution which inevitably suffered from sampling bias (e.g., preponderance of females in our samples) and hence possible lack of generalisability to the general population.

\section{Conclusion}

Chronic constipation is a common health problem especially affecting the older people. We hereby presented the use of a novel numeric scoring system as we performed a cross-sectional retrospective analysis of bowel habits and the use of laxatives amongst residents of a long term care facility in Kingston. Our pilot data suggested that our novel numeric stool scoring system maybe a useful tool to enable quantitative assessment of the stool texture in relation to the stool frequency and enable comparison between groups within a specified time. Larger prospective trials are needed to further test our proposed stool scoring system.

\section{Conflict of Interests}

None

\section{Contribution}

RW was the PGY2 resident who carried out the study as her own resident research project and contributed to the data analysis and writing of the manuscript. LL is Associate Professor and conceived the idea of the stool scoring system and the design of the study, performed the majority of data analysis and wrote the manuscript.

\section{References}

1. Drossman DA, Sandler RS, McKee DC, Lovitz AJ (1982) Bowel patterns among subjects not seeking health care. Use of a questionnaire to identify a population with bowel dysfunction. Gastroenterology 83: 529-534.

2. Connell AM, Hilton C, Irvine G, Lennard-Jones JE, Misiewicz JJ (1965) Variation of bowel habit in two population samples. Br Med J 2: 1095-1099.

3. Pare P, Ferrazzi S, Thompson WG, Irvine EJ, Rance L (2001) An epidemiological survey of constipation in canada: definitions, rates, demographics, and predictors of health care seeking. Am J Gastroenterol 96: 3130-3137.

4. Heaton KW, Radvan J, Cripps H, Mountford RA, Braddon FE, et al. (1992) Defecation frequency and timing, and stool form in the general population: a prospective study. Gut 33: 818-824.

5. Lewis SJ, Heaton KW (1997) Stool form scale as a useful guide to intestinal transit time. Scand J Gastroenterol 32: 920-924.

6. McCrea GL, Miaskowski C, Stotts NA, Macera L, Varma MG (2009) A review of the literature on gender and age differences in the prevalence and characteristics of constipation in North America. J Pain Symptom Manage 37: 737-745.

7. Higgins PD, Johanson JF (2004) Epidemiology of constipation in North America: a systematic review. Am J Gastroenterol 99: 750-759.

8. Bouras EP, Tangalos EG (2009) Chronic constipation in the elderly. Gastroenterol Clin North Am 38: 463-480.

9. Hosia-Randell H, Suominen M, Muurinen S, Pitkälä KH (2007) Use of laxatives among older nursing home residents in Helsinki, Finland. Drugs Aging 24: $147-154$

10. Brandt LJ, Prather CM, Quigley EM, Schiller LR, Schoenfeld P, et al. (2005) Systematic review on the management of chronic constipation in North America. Am J Gastroenterol 100: S5-5S21.

11. McCrea GL, Miaskowski C, Stotts NA, Macera L, Paul SM, et al. (2009) Gender differences in self-reported constipation characteristics, symptoms, and bowe and dietary habits among patients attending a specialty clinic for constipation. Gend Med 6: 259-271.

12. Choung RS, Locke GR 3rd, Schleck CD, Zinsmeister AR, Talley NJ (2007) Cumulative incidence of chronic constipation: a population-based study 19882003. Aliment Pharmacol Ther 26: 1521-1528.

13. Shaheen NJ, Hansen RA, Morgan DR, Gangarosa LM, Ringel Y, et al. (2006) The burden of gastrointestinal and liver diseases, 2006. Am J Gastroenterol 101: $2128-2138$

14. Rantis PC Jr, Vernava AM 3rd, Daniel GL, Longo WE (1997) Chronic constipation--is the work-up worth the cost? Dis Colon Rectum 40: 280-286.

15. Nyrop KA, Palsson OS, Levy RL, Korff MV, Feld AD, et al. (2007) Costs of health care for irritable bowel syndrome, chronic constipation, functional diarrhoea and functional abdominal pain. Aliment Pharmacol Ther 26:237-248.

16. Wald A, Scarpignato C, Kamm MA, Mueller-Lissner S, Helfrich I, et al. (2007) The burden of constipation on quality of life: results of a multinational survey. Aliment Pharmacol Ther 26: 227-236.

17. O’Keefe EA, Talley NJ, Zinsmeister AR, Jacobsen SJ (1995) Bowel disorders 
Citation: Wijeratne R, Leung L (2013) Use of a Novel Stool Scoring System for Assessing Bowel Habits and Laxatives use Amongst Older People-A Pilot Study in Kingston, Ontario. J Gen Pract 1: 124. doi: 10.4172/2329-9126.1000124

Page 4 of 4

impair functional status and quality of life in the elderly: a population-based study. J Gerontol A Biol Sci Med Sci 50: M184-189.
18. Leung L, Riutta T, Kotecha J, Rosser W (2011) Chronic constipation: an evidence-based review. J Am Board Fam Med 24: 436-451. 